\title{
Werner Sollors Zur Rassenjustiz in den U.S.A.
}

\begin{abstract}
We believe that all bladk people should be released from the many jails and prisons because they have not received a fair and impartial trial.

We believe that the courts should follow the United States Constitution so that black people will receive fair trials. The 14 th Amendment of the U.S. Constitution gives a man a right to be tried by his peer group. A peer is a person from a similar economic, social, religious, geographical, environmental, historical and racial background. To do this the court will be forced to select a jury from the blads community from which the black defendant came. We have been, and are being tried by all-white juries that have no understanding of the saverage reasoning man* of the bladk community.
\end{abstract}

(Aus dem Programm der Black Pantbers)

I.

*Von echtem Rassenrecht [das Unterscheidungen nicht auf Grund von sekundären Merkmalen trifft] kann heute im wesentlichen nur in drei Ländern der Welt die Rede sein: In den Vereinigten Staaten von Amerika, im Deutschen Reich und - schon in weit schwächeren Ansätzen - in der Südafrikanischen Union. \&'

Diese Bemerkung entstammt einer in den dreißiger Jahren mit Hilfe der Deutschen Forschungsgemeinschatt entstandenen Arbeit über Das Rassenrecht in den Vereinigten Staaten. Der Autor schränkt diese Feststellung mit seinem Bedauern über die Unzulänglichkeit und mangelnde Einheitlichkeit des amerikanischen Rassenrechts ein. $\mathrm{Zu}$ dieser rassistischen Klage hätte er heute viel eher Anlaß als 1936.

Der fortschreitende gesetzliche Abbau des »echten Rassenrechts «, besonders seit der Entscheidung des Obersten Gerichrshofes von 1954, hat die rechtliche Situation der Afro-Amerikaner geändert. In der Praxis freilich bleiben sie auch nach der Bürgerrechtsgesetzgebung dem weißen Rassismus ausgeliefert. In Vietnam werden die Afro-Amerikaner von der »integrierten « Armee vor allem in den unteren Rängen an den Kampflinien eingesetzt, so daß sie zwischen einem Viertel und einem Siebtel der Gefallenen stellen, obwohl sie insgesamt nur ein Zehntel der in Vietnam interverierenden US-Truppen ausmachen. ${ }^{2}$ In ihrem ${ }^{2}$ Stiefmutterland « Amerika stehen Polizeiaktionen gegen Einzelpersonen und gegen Gruppen, ja militärische Einsärze gegen ganze Stadtteile, an der Tagesordnung und haben seit r96s ca. 200 Todesopfer gefordert. Die Zahl der Verhaftungen

1 Heinrich Krieger, Das Rassenrecht in den Vereinigen Staaten, Neue Dt. Forschungen, Abt. Staats-, Verwaltungs-, Kirchen-, Völkerrecht und Staatstheorie Bd. 6 (Berlin, s936), p. ro.

2 Vgl. dazu Thomas A. Johnson, $>$ Negroes in the Name, Ebony (August, 1968). In der gleichen Ausgabe der Zeitschrift auch ein Artikel über Rassismus in der Bundesrepublik. 
steigt ständig. 1964 wurden 4381419 Menschen in den USA verhaftet, davon I 194377 Afro-Amerikaner. ${ }^{3} 29000$ Verhaftungen gab es allein bei den GettoUnruhen von 1965 bis Ende 1967 ; und nur in den Wochen nach der Ermordung Martin Luther Kings waren es erneut $28271 .^{4}$.

Die seit Ende des vorigen Jahrhunderts erlassenen Segregationsgesetze werden zunehmend außer Kraft gesetzt, und die Bestrafung von "Delikten $\propto$ wie dem Besuch eines weißen Lokals oder der Mischehe (die 1936 in 30 Staaten und 1967 "nur noch « in 17 Staaten strafbar war ${ }^{5}$ ) geht zurück. Bestehen bleiben die weiBen Geschworenengerichte, die sich nach angelsächsischer Tradition aus Gleichen, "peers« der Angeklagten zusammensetzen sollten. Bestehen bleibr ferner die Tendenz vieler weißer Gerichte, Afro-Amerikaner exemplarisch, d. h. besonders hart zu bestrafen. Davon sind vor allem diejenigen Afro-Amerikaner betroffen, die zum Verteidigungskampf gegen alle Formen des weißen Rassismus angetreten sind, die also auch Elemente von Rassenjustiz bloßstellen. Sie geraten unausweichlich in ein sich ständig ausweitendes Netz von Ordnungsstrafen, Nebenverfahren, überhöhten Kautionen, Abhöraffären und erhalten in den Hauptverfahren unter formalen Vorwänden in der Regel die Höchststrafen, die freilich weniger dem betreffenden Tatbestand gelten als ihrer Hautfarbe und ihrem politischen Kampf.

Viele Weiße, darunter viele weiße Richter, können sich von ihrem ambivalenten Negerbild niche befreien und sehen in ihrem schwarzen Angeklagten nur entweder den etwas dümmlichen »boy" oder, wenn er sich diesem Bild nicht fügt, den elementaren, tierischen, gefährlichen Täter. Eine liberale Frankfurter Dissertation der zwanziger Jahre macht unfreiwillig diese Denkstruktur deutlich, die in der Bundesrepublik ebenso wie in den Vereinigten Staaten weiterlebt:

Zum großen Teil finder die Rassenabneigung ihre Begründung in der Natur des Negers. Die Eigenschaften seiner afrikanischen Stammesgenossen hat der Neger im allgemeinen auch in Amerika beibehalten. Vor allem ist es das Außere und die Hautausdünstung, die dem Weißen den Umgang mit dem schwarzen Mann unangenehm macht. Dazu wirft er ihm recht üble Charaktereigenschaften, Sinnlichkeit, Energie- und Zügellosigkeit, Mangel an Wahrheitsliebe und Verantwortungsgefühl, Freude an Lärm und Faulheit vor. $\mathrm{Daß}$ der Neger dabei aber auch ein gurmütiger, nachgiebiger, unterwürfiger und hilfsbereiter Mensch ist, der seine Dienertreue oft bewiesen hat, wird meist weniger beachtet. ${ }^{\circ}$

Der Frankfurter Rechtsanwalt, der vor einigen Jahren das Gericht bat, die Emotionalität seines farbigen Mandanten als mildernden Umstand zu berüdssichtigen, wäre sicher ebenso über einige Negerprozesse erschrocken wie seine - ähnlichen Klischees verhafteren - amerikanischen Juristenkollegen. Wo afroamerikanische Angeklagte ihren Richtern intellektuell überlegen sind, müssen sie mit der Höchststrafe rechnen. Sind sie "gutmütig», beharren aber auf ihrem Rechtsstandpunkt, erwartet sie - wohl wegen ihrer Unbelehrbarkeit - die gleiche Strafe. Geben sie schlagfertige Antworten, so erhalten sie zur Höchststrafe noch Ordnungsstrafen. So wird ihre Abweichung vom Bild des treuen Dieners geahndet. Einige Fallentwicklungen können diese Behauptungen illustrieren.

\footnotetext{
3 Lt. Discrimination in Law Agencies, the Courts and Penal Instirutions, in Negro Handbook, hrsg. v. Ebony (Chicago, 1966), pp. 102-111.

4 It. Guardian (London) v. 2. II. 1967 und Tagesspiegel v. 23. 4. 1968.

5 Vgl. The Legal Status of the Negro, * in The Negro Almanac, hrsg. v. Harry A. Ploski u. Roscoe C. Brown, Jr. (New York, 1967), pp. I87-215.

- Kurr Weimar, Die Negerfrage in den Vereinigten Staaten von Amerika (Frankfurt, 1929), p. ss.
} 


\section{Blad People!}

What about that bad short you saw last week on Frelinghuysen, or those stoves and refrigerators, record players, shotguns, in Sears, Bambergers, Klein's, Hahnes', Chase, and the smaller joosh enterprises? What about that bad jewelry, on Washington Street, and those couple of shops on Springfield? You know how to get it, you can get it, no money down, no money never, money dont grow on trees no way, only whitey's got it, makes it with a machine, to control you you cant steal nothin from a white man, he's already stole it he owes you anyching you want, even his life. All the stores will open if you will say the magic words. The magic words are: Up against the wall mother fucker this is a stick up! Or: Smash the window at night (these are magic actions) smash the windows daytime, anytime, together, lets smash the window drag the shit from in there. No money down. No time to pay. Just take what you want. The magic dance in the street. Run up and down Broad Street niggers, take the shit you want. Take their lives if need be, but get what you want what you need. Dance up and down the streets, turn all the music up, run through the streets with music, beautiful radios on Marker Street, they are brought here especially for you. Our brothers are moving all over, smashing at jellywhite faces. We must make our own World, man, our own world, and we can not do this unless the white man is dead. Let's get together and kill him my man, lets go to gather the fruit of the sun, let's make a world we want black children to grow and learn in do not let your children when they grow look in your face and curse you by pirying your tomish ways.

Als Jones dieses Gedicht am 3. Oktober 1966 im Village Theatre in New York vortrug, spitzte die New York Times in ihrer Berichterstatrung den ohnehin aggressiven Ton des Gedichts noch zu: „our brothers are moving all over, smashing at jellywhite faces" wird in der Zeitung zu: "Smash their jellywhite faces." Wo sinngemäß richtig zitiert wird, folgt keine Auseinanderserzung mit dem politischen Hintergrund des Gedichts, sondern der Versuch, mit der Darstellung der Reaktion einiger weniger Zuhörer (die den Saal verließen) die politischen Implikationen zu bagatellisieren. Der Angriff auf die ungerechten Besitzverhältnisse, der diesem Gedicht seine Substanz gibt, wird nicht ernst genommen, sondern lediglich als typischer Ausdruck des ohnedies notorischen Polemikers Jones verstanden: $\bowtie$ Er erhielt gestern abend seinen Ruf aufrecht«, "gab seinen Lieblingsthemen Ausdruck «. Diese Freiheit, »seine Lieblingsthemen« zu äußern, fand ihre Grenze, als Jones auch im Privatleben mit der politischen Bewegung identifizierbar wurde, auf die seine Gedichte sich nur bezogen hatten.

Am I4. Juli I967, während des Getto-Aufstandes in Newark, wurde LeRoi Jones von Polizeischlägen verletzt und mit zwei Freunden verhaftet, weil er, nach Polizeiangaben, zwei Pistolen in seinem VW-Bus verstedkt hatte. Es fiel der New York Times leicht, mit Hilfe modifizierter Archiv-Informationen eine nachträgliche Rechtfertigung der Polizei-Maßnahme zu liefern, der es an formaler Glaubwürdigkeit fehlte. "Letztes Jahr bezog er sich in einem Vortrag auf die Weißen «, heißt es in der Zeitung, sund riet den Negern, ihnen die geleeweißen Gesichter einzuschlagen «. Aus dem Gedichtabend, den man noch tolerieren konnte, solange der Dichter seine Angriffe auf die weiße Machtstruktur allein in Gedichtform vortrug, wurde nun rückwirkend ein Vortrag, der LeRoi Jones in Verbindung mit Malcolm X oder Robert F. Williams brachte. Denn wie 
in den Berichten über diese wird auch hier verschwiegen, wie die Notwendigkeit der Selbstverteidigung begründet ist und wie die Gesellschaft aussieht, in der Gegengewait entstehen muß. Vielmehr haftet Jones von nun an - aufgrund einer falsch zitierten Gedichtzeile - der Vorwurf an, daß er ein »Hasser« sei, der »Gewalttätigkeit befürworte

Von hier ist es nur ein kleiner Schritt zur unverhohlenen Schadenfreude, daß wieder einmal ein *Befürworter der Gewalt« zum Opfer nicht der Polizeibrutalitït, sondern seines eigenen Prinzips geworden sei. Im Falle von Malcolm X hatte die New York Times diesen schadenfrohen Gedankengang bereits vorexerziert und nach dessen Ermordung in einem Kommentar ausgeführt: "Sein... fanatischer Glaube an die Gewalt prädestinierte ihn für Ruhm und für ein gewaltsames Ende. " Dazu hat George Breitman treffend bemerkt: $»$ Damit wird sein angeblicher Glaube mit seinem Tod in eine Art Kausalbeziehung gesetzt; er war für seine eigene Ermordung verantwortlich. « Analog dazu heißt es über Jones in TIME Magazine:

Jones, der den Negern einmal sagte, die einzige Art, mit den Weißen ferrig zu werden, sei ihnen, die weißen Geleegesidhter einzuschlagen, wurde nun selbst zusammengeschlagen; eine stumpfe Waffe verletzre seinen Schädel und verursachte eine Wunde, die mit sieben Stichen genäht werden mußte.

Diese von der Presse stimulierte „Gedichtinterpretation « sollte im weiteren Verlauf des Prozesses noch eine wichtige Rolle spielen. Doch zunächst zurück zum Fall selbst. Die Polizeidarstellung des Sachverhalts ist leicht zugänglich, wurde sie doch von New York Times, TIME und Newsweek übernommen:

Newark, 14.7. 1967-LeRoi Jones, der 32-jährige Dramatiker, Dichter und Jazzkritiker, erlits heute eine Kopfverletzung und wurde mit der Begründung verhafter, Waffen in einem von Unruhen betroffenen Viertel Newarks mit sich geführt zu haben. Die Polizei gab an, daß Jones ... mit einer Flasche am Kopf getroffen worden sei. Es sei unbekannt, wer die Flasche geworfen habe.

Jones wurde im städtischen Hospital behandelt und später von der Polizei unter Anschuldigung des Waffentragens mitgenommen. Die Polizei gab an, er hätte zwei geladene 32er Pistolen in seinem Besitz gehabt.

Der bärtige Schriftsteiler, der seine heftig anti-weißen Anschauungen schon häufig zum Ausdrudk brachte, wurde ... nicht beschuldigt, die Waffen benutzt zu haben.

Nach dem Polizeibericht wurde Jones mit zwei andern Männern in einem grünen VWBus an der Ecke South Orange Avenue und Seventh Street angehalten ...

Die Polizei gab an, daß eine Warnung vor drei Männern in einem solchen Fahrzeug ausgegeben worden war, da sie beim Schießen mit Pistolen beobachtet worden waren ... ${ }^{7}$

Die Darstellung der Verhafteten ist schon schwerer auffindbar. Sie wurde im Zusammenhang hauptsächlich von kleineren oder linken Zeitschriften publiziert:

Wir waren auf dem kürzesten Weg nach Haus, als wir von zwei Mannschaftschaftswagen von Polizei mit Helmen und Pistolen... angehalten wurden... Wir mußten aus dem Auto aussteigen... Ein Zivilbeamter, den ich als Schulkameraden von der Harringer High School wiedererkannte, rief, daß wir die $»$ Bastarde $\propto$ wären, die auf sie geschossen hätten. Ja*, rief er, »ein blauer Lastwagen mit Plane.* (Ich habe einen olivgrünen VW-Bus). Id sagte, daB wir auf niemanden gesctossen härten. Dem Zivilbearnten sagte

7 Um den Fußnotenapparat klein zu haiten, erfolgen bei übersetzten Zeitungs- und Zeitungsberichten keine weiteren Angaben. Sie entstammen, wenn nidit anders angegeben, der New York Times des jeweiligen Tages. 
ich, daß ich ihn noch von der Schulzeit her kenne - worauf er mich ins Gesicht schlug und gegen das Auto schleuderte.

Dann stieß er mich so fest er konnte mit seiner Pistole in den Bauch und fragte, wo die Waffen wären. Ich sagte $i h m, d a ß$ wir keine Waffen härten. Plötzlich schien es, daß fünf oder sechs Polizisten mich umringten und mich zu schlagen begannen. Ich wurde ungefähr fünf mal von ihren Stöcken auf meinen Kopf getroffen ... Als ich hinfel, versuchte ein Polizist, mir in den Unterleib zu treten... Während sie mich schlugen, nannten sie mich "Tier und fragten mich nach den Waffen. Im Wagen ging das Schlagen weiter... Im Polizeipräsidium stand Herr Spina, der Polizeipräsident, hinter dem Tisch. Idh fragte ihn, ob er angeordnet hätte, daß ich zusammengeschlagen würde. Er antwortete, ndich haben sie erwischt, was? * - mit einem Lächeln ...

Dann wurden wir ins städrische Hospital gebrachs. Ich wurde ... mit Handschellen an einen Rollstuhl gefesselt. Die »Arzte « nähten meine Wunde mir acht oder neun Stichen, und ein Doktor rief mir zu: $\bullet$ Du bist ein Dichter? $\mathrm{Na}$, du wirst für eine ganze Zeit keine Gedichre schreiben!« Zu keiner Zeit durften wir telefonieren oder uns über unsere Rechrslage informieren...8

Nach der mehrtägigen Untersuchungshaft, aus der Jones gegen eine Kaution von 25000 Dollars entiassen wurde, war er überzeugt, daß der Prozeß nicht gerechter sein würde als die Polizeimaßnahme und die Wundbehandlung. Er meinte, "das sogenannte Gerichtssystem in Newark ist ebenso korrupt und rassistisch wie die Polizeibehörde». Tatsächlich schenkten die (weißen) Geschworenen auch dem Polizeibericht Glauben, und Jones versuchte nur noch, den Scheincharakter des Verfahrens mit rhetorischen Mitteln bloßzustellen. Er weigerte sich am ersten Verhandlungstag sich hinzusetzen und bestand darauf, eine Erklärung abzugeben. Der Richter Leon W. Kapp sagte daraufhin: „Das ist äußerst regelwidrig. Dies ist ein Ort der Rechtsfindung. " Jones: "Dies ist kein Ort der Rechtsfindung. Ich habe Ihre Meinung schon gestern gelesen, Sie sind disqualifiziert. "Dann wies Jones mit seinem Arm auf die Geschworenen und rief: »Ich werde mich nicht von 100 weißen Leuten richten lassen. Sie sind nicht meine peers, sie sind meine Unterdrüdker ... Ich habe hier nichts verloren. "Für seinen Versuch, den Gerichtssaal zu verlassen, und seine Bemerkung, er stehe vor einem »kangaroo court《 (ein Slang-Ausdruck für ein Richter-Spiel unter Strafgefangenen) erhielt er eine Ordnungsstrafe von dreißig Tagen, die von der nächsten Instanz allerdings - vorläufig - aufgehoben wurde.

Tatsäcalich wurde auf Antrag der Verteidigung der Tagungsort nach Morristown (New Jersey) verlegt, wo der Sheriff Arnold Ladd 77 Bürger auf der Straße anheuerte, von denen 12 (Weiße) die Jury bildeten. Am 7 . November I 967 fanden sie Jones nach 75-minüriger Beratung schuldig, nicht ohne Beeinflussung durch den Richter, der die Polizei stets für glaubwürdiger hielt und voller Stolz die Polizeizeugen "these five men in blue « nannte. "Denn schließlich ist der Polizeibeamte ja der Schurz der Gemeinschaft gegen Gewaltanwendung und andere Gesetzwidrigkeiten.«

Jones wurde zwar die Unterstützung der Bürgerrechtsorganisation CORE (Congress of Racial Equality) zuteil, die am Fall Jones Rechtsmittel gegen die Institution des weißen Geschworenengerichts einlegte, doch lief der Prozeß zunächst weiter, und LeRoi Jones wurde am 4 . Januar I 968 zur Höchststrafe von $21 / 2$ bis 3 Jahren Gefängnis und einer Geldstrafe von rooo Dollars verurteilt. Der Verlauf der Verhandlung und die Urreilsbegründung verdienen ausführlicher dargestellt zu werden.

8 Aus einem Leserbrief Allen Ginsbergs an Evergreen Review, 7. 12, n. 53, p. 12. In der gleichen Zeitschrift, v. 12, n. 50, p. 49 ist das Gedicht $\times$ Black People! $\ll$ abgedrudkr. 
Zunächst las Richter Kapp nahezu den gesamten Mittelteil des Gedichts »Bladk People!« vor, wobei er alle anstößigen Wörter durch »blank« ersetzte. Dann deutete er an, daß dieses Gedicht (in der Berichterstattung der Londoner Times wiederum zum "Arrikel« geworden), das einen Monat vor dem Gerichtstermin in der Zeitschrift Evergreen Review abgedrudkt worden war, zur Härte des Urteils beirrüge.

K. Dieses diabolische Rezepe zum Morden, Stehlen und Plündern errege Verdacht, daß Sie an der Planung beteiligt sind, den Funken zu zünden und Newark in Flammen zu serzen... Es ist meine wohlüberlegre Überzeugung, daß Sie krank sind und medizinische Behandlung benötigen ...

J. Nicht so krank wie Sie!

K. Ihre Begabung ist irregeleitet worden. Sie haben die Fähigkeit, einen gesunden Beitrag zur Beilegung bestehender Spannungen zu leisten. Stattdessen sehen wir Sie in der Avantgarde einer extremradikalen Gruppe von Befürwortern der Zerstörung ...

J. Der Zerstörung der Ungerechtigkeir!

K. ... unserer demokratischen Grundordnung. Wenn der Philosoph sein eigenes Gesetz machen darf, so auch der Narr.

J. Das sehen wir ja.

K. Das Urteil dieses Gerichtes lautet wegen unerlaubten Besitzes von zwei Revolvern ...

J. ... und zwei Gedichten...

K. ... folgendermaßen: Sie sind zu nicht weniger als $2^{1 / 2}$ und nicht mehr als 3 Jahren Freiheitsentzug ... und einer Geldstrafe von roo0 Dollars verurteilt.

J. Nur keine Sorge, Bruder Kapp, die Schwarzen werden mich schon richten. Sie repräsentieren den Willen einer zerbrödkelnden Struktur, und ich bin ein freier schwarzer Mann.?

Auf das Urteil folgte eine Welle verbaler Proteste. Zuerst reagierten die Dichter Corso, Ginsberg, Olson, Mc Clure, Duncan, Wieners und Ferlinghetri. In einem Brief vom 6. Januar 1968 nannten sie Jones einen sbewußten amerikanischen Künstler, der für seine Dichtung ... ins Gefängnis soll.« P.E.N. und Civil Liberties Union kritisierten als nächste die Verletzung von Jones' Recht auf freie Meinungsäußerung.

Tatsächlich ist der Fall aber anders gelagert und ein Modell für die Grenzen der Freiheit nicht so sehr der Kunst als des afro-amerikanischen Künstlers in den Vereinigten Staaten der Gegenwart. Die Zensur der literarischen Publikationen ist stark zurüdkgegangen, und gerade sexuell und politisch *schockierende* Literatur kann nicht nur in den - teilweise staatlich subventionierten - »Underground «-Organen ungehindert erscheinen. Die Autoren werden für ihren »Mut « gelobt und mit werbenden Markenzeichen »wütend«, »zornig« oder »schockierend « belegt. Das ist jedoch ihrer Integration in die Kulturindustrie eher zuträglich, in deren Maschinerie potentiell gefährliche Aspekte der Werke neutralisiert werden. Gesellschaftskritisches wird entpolitisiert und oft in psychologische Betrachtungen des Autors umgemünzt. Die Literatur allein ist harmlos geworden; die Wirkung der einstmals verbotenen Früchte von James Joyce und Henry Miller kann von Büchern allein in Amerika nict mehr ausgehen. Auch militanteste Literatur ist eben als »militante Literatur « integriert und konsumierbar. Gefährlich kann heute ein Autor nur werden, wenn er die Gesellschaft mit diesem Integrationsbetrieb angreift, und zwar nicht mehr nur in der Literatur, sondern auch im persönlichen Engagement.

Es ist bezeichnend, daß der Richter Kapp offenbar nie daran dachte, die Num-

- Vgl. Newsweek v. Is. I. 1968, dem der größte Teil des Urteilsdialogs entnommen ist. 
mer von Evergreen Review verbieten und einziehen zu lassen, die das beanstandete Gedicht enthielt, oder daß die New York Times in ihrem ersten Bericht über die Dichterlesung von $»$ Bladk People! nicht impliziert, daß solche Leseabende nicht stattfinden sollten. Das Kunstprodukt bleibt unangetastet, weil es selbst nichts mehr antasten kann; der Künstler jedoch wird unter Vorwänden, die rechtlich nicht stichhaltig sind, gemaßregelt. Er hat die Möglichkeit, wieder zu seinem »Beruf« zurüdkzukehren und nur Künstler zu bleiben oder wieder zu werden.

Im Dezember r 968 wurde dem Revisionsantrag der Verteidigung stattgegeben, da feststand, daß der Richter die Geschworenen zu beeinflussen versucht hat. Eine Neuauflage des ganzen Prozesses steht bevor. ${ }^{90}$

\section{Mubammad Ali, Boxer, geb. 1942}

Nach den sportichen Erfoigen auf der Olympiade 1960 machte Cassius Marcellus Clay eine steile Karriere als Boxer. Am 25. Feburar 1964 wurde er nach seinem Sieg über Sonny Liston Weltmeister in der Schwergewichtsklasse. Im selben Jahr noch nahm er den Namen Muhammad Ali an, da er, wie alle Mitglieder der Nation of Islam, seinen englischen Namen als Sklavennamen ablehnt. Seine Erfolge als Boxer (er war so gut, daß die Agenturen schon von Schwierigkeiten redeten, überhaupt noch Gegner für ihn zu finden) verhalfen der Nation of Islam zu Popularität und größerer Mitgliedschaft. Das Programm der afroamerikanischen Muslims sieht u. a. die Schaffung schwarzen Selbstbewußtseins durch Isolation von der weißen Gesellschaft vor. In der $Z$ wischenzeit fordern sie in ihrem Credo:

We want justice. Equal justice under the law. We want justice applied equally to all, regardless of creed or class or color. ${ }^{10}$

Diese Forderung ist viel weniger spezifisch als etwa die des eingangs zitierten Black Panther-Programms. Die Floskel "regardless of creed « nimmt jedoch eine besondere Bedeutung ein, wenn es in ihrem Credo weiter heißt:

Wir glauben nicht, daß diese Nation uns dazu zwingen sollte, an ihren Kriegen teilzunehmen; denn wir haben nichts daran zu gewinnen, solange uns Amerika nicht ein eigenes Territorium bewilligt, in dem wir etwas zu verteidigen haben werden.

Dieser Glaubensartikel wurde vor allem während der Zeit der extensiven Einberufung von Afro-Amerikanern für den Krieg gegen das vietnamesische Volk ernst genommen. Muhammad Ali hatte zunächst keine Schwierigkeiten, da er in seiner Heimatstadt Louisville (Kentudky) als $\mathrm{I} / \mathrm{Y}$ gemustert worden war; d. h. er galt als körperlich tauglich, aber nicht intelligent genug, um eingezogen zu werden. Mit der Eskalation des Krieges wurden jedoch auch die Musterungsverordnungen geändert. Das wurde am ro. Februar r 966 in einer Pressemeldung bekanntgegeben, die besonders auf schwarze Athleten anspielt und Clay namentlich aufführt.

Am I 8. 2. 1966 erfuhr Clay in Miami von einem gut informierten Reporter, daß die Erfassungsbehörde in Louisville seine Klassifizierung geändert, und er nun

Q" Jones ist inzwischen in zweiter Instanz freigesprochen worden.

10 Das Credo der Nation of Islam ist auf der Rijdkseite jeder Ausgabe ihres Publikationsorgans Muhammad Speaks abgedrudkt. 
den I/A Status erhalten habe, d. h. mit der baldigen Einberufung rechnen müß-

te. Clay sagte daraufhin vor der Presse:

Wie können die mich plötzlich I/A einstufen. Wie können sie ohne einen neuen Test herausfinden, ob ich doofer oder besser bin als letztes Mal? Zwei Jahre lang hat mich diese Regierung vor der ganzen Welt in Verlegenheit gebracht, weil die Leute denken mußten, daß ich ein Blödmann bin. Das hat mich ganz schön nervös gemacht, und meine Mutter und mein Vater haben gelitten. Und nun machen sie mich so über Nacht zum I/A ohne Test oder irgendwas Offizielles.

Von Journalisten nach seiner Meinung über den Vietnamkrieg gefragt, soll er geantwortet haben:

Ith bin ein Mitglied der Nation of Islam, und wir gehen in keine Kriege, die Allah nicht selbst erklärt hat. Mit dem Vietkong habe ich keinen persönlichen Streit.

Kaum hatte er die Bemerkung »I don't have no personal quarrel with those Vietcongs « gemacht, hatte er die ersten Schwierigkeiten. Seine angesagten Boxkämpfe wurden von Governor Otto Kerner (Illinois) und Bürgermeister Richard Daley in Chikago verboten, falls Muhammad Ali sich nicht für seine »unpatriotischen« Außerungen entschuldigen würde. Ali entschuldigte sich nicht und mußte von nun an seine Boxkämpfe fast ausschließlich im Ausland austragen; selbst ein Indianerreservat wurde ihm als Austragungsort verweigert.

Bald nahm sich Muhammad Ali einen Spezialanwalt für Kriegsdienstfragen, der im zweiten Weltkrieg Zeugen Jehovas rechtlich vertreten hatte, und versuchte als Verweigerer aus Gewissensgründen, und später als Geistlicher der Nation of Islam die Einberufung zu umgehen. Der Preis "Boxer des Jahres « wurde ihm daraufhin entzogen. Doch verliefen alle Versuche, den Einberufungstermin aufzuschieben, ergebnislos. Auch die berechtigte Klage gegen die mangelnde Vertretung von Negern in der Musterungsbehörde wurde vom Obersten Bundesgericht abgewiesen. Seine Einberufung war auf den 28. April 1967 festgesetzt. Muhammad Ali sah keinen anderen Ausweg, als bei der Einberufung zwar zu erscheinen, aber den Eid zu verweigern. Er sagte am 20. 4. 1967: \$Ich weiß, was ich dafür kriege: fünf Jahre und ro 000 Dollar - das ist alles.« Und er fragte weiter:

Warum fragen sie gerade mich, einen sogenannten Neger, eine Uniform anzuziehen und Io 000 Meilen von zu Haus entfernt Bomben und Kugeln auf braune Vietnamesen loszulassen, während den sogenannten Negern hier in Louisville die Menschenrechte verweigert werden und sie wie Hunde behandelt werden? Ich will weder meine Religion noch mein Volk oder mich selbst entehren und als Werkzeug zur Versklavung derjenigen dienen, die für Gerechtigkeit, Gleichheit und Freiheit kämpfen.

Als Muhammad Ali diese Außerungen machte, war die extensive Einberufung, zumal von Afro-Amerikanern, für die Kriegführung unerläßlich geworden. So war die öffentliche Reaktion auf seine Entscheidung entsprechend hart, und das Justizministerium wies auch einen Antrag ab, ihn nicht als Verletzer der Kriegsdienstgesetze zu melden, wenn er sich nur bei der Einberufung einstellt, den Eid jedoch verweigert. Die Begründung Fred Drogulas, des Rechtsanwalts aus dem Justizministerium, lautet: $»$ Wenn er gewinnt, werden alle Muslims verweigern, und woher sollen wir dann Soldaten kriegen? «

Als Muhammad Ali am 28.4. I 967 bei der Einberufungsbehörde in Houston (Texas) tatsächlich den Eid verweigerte, wurde ihm binnen zweier Stunden der 
Weltmeisterschaftstitel der World Boxing Association abgesprochen; und ein Verfahren wegen »draft evasion « wurde gegen ihn eingeleiter. Der Ton, mit dem die New York Times seine Entscheidung kommentiert, ruft die Angst des Establishments nach den großen Anti-Vietnamkriegsdemonstrationen in Erinnerung.

Bürger können nichr aussuchen und wählea, welche Kriege sie kämpfen möchren; ebensowenig können sie sich die Gesetze aussuchen und wählen, die sie befolgen möchten. Wenn jedoch Cassius Clay und andere Verweigerer im Einberufungsalter glauben, da B der Krieg in Vietnam ungerecht ist, haben sie die Wahl, für ihren Glauben ins Gefängnis zu gehen... Das Risiko ist geringer als für die jungen Männer, die gern für ihr Land in Vietnam und in anderen gefährlichen Gegenden kämpfen.

Wenn selbst eine liberale Zeitung des Landes, in das einst europäische Kriegsdienstverweigerer flüchteten, zu diesem Zynismus bereit war, konnte man vom Gericht in Houston (dessen I 2 Geschworenen kein Neger angehörte) keine Milde erwarten. Die Tatsache, daß Martin Luther King und Stokely Carmichael die Entscheidung Muhammad Alis begrüßten, konnte das texanische Gericht ebenfalls nicht umstimmen: am 2r. Juni 1967 wurde Muhammad Ali zur Höchststrafe von fünf Jahren Gefängnis und ro 000 Dollar Geldstrafe verurteilt. Seine Gutwilligkeit, überhaupt zum Einberufungsort zu kommen, wurde nicht honoriert. Wie im Falle von LeRoi Jones griff die Jury aus Gründen, die nicht zur Anklage gehörten, zur Höchststrafe. Wie im Falle von Jones wird jedoch auch bei Muhammad Ali noch über eine Revision entschieden.

\section{$4 \cdot$}

Die beiden so ausführlich geschilderten Fälle sind keine Ausnahme. Die Angst vor Unruhen, vor Beispielen von Civil Disobedience für die zwanzig Millionen Afro-Amerikaner, verführt weiße Geschworenengerichte immer wieder zum exemplarischen Bestrafen. H. Rap Brown, der 1943 geborene ehemalige S.N.C.C. (Student Non-Violent Coordinaring Committee)-Vorsitzende, steht in einer ganzen Anzahl von Verfahren, die ihm bisher die Höchststrafe für unerlaubren Transport einer Waffe von einem Staat in einen andern (fünf Jahre Gefängnis; ein Berufungsverfahren läuft), und Kautionen bis zu 100000 Dollars auferlegt haben. Wie im Falle von Jones, brachten Presse und Anklage eine Schußverletzung an seinem Kopf mit einer feurigen Rede von ihm in einen Kausalzusammenhang (Cambridge, Maryland, 25. Juli 1967). Erst später mußte die Polizei zugeben, daß sie von der Schußwaffe Gebrauch gemacht hat. $D a$ es am Tage von Browns Rede auch zu Unruhen und einer Brandstiftung kam, steht er - auf Mitiniriative des damaligen Gouverneurs von Maryland, Spiro T. Agnew, hin - unter der Anklage der Anstiftung zur Brandstittung und zum Aufruhr; und das in einer Stadt, die schon $1964 / 1965$ fast ein ganzes Jahr lang von der Miliz besetzt war, und deren Feuerwehr zugeben mußre, daß sie an dem betreffenden Abend, "unter anderem aus Rache" ihren Einsatz mehrere Stunden hinausgezögert hatte.

Ebenso sind noch zahlreiche Verfahren gegen Stokely Carmichael und viele Mitglieder der Black Panthers im Gange. Wie bei Muhammad Ali werden bei vielen von ihnen Telefon und Post überwacht, wie Rap Brown wird ihnen gelegentlich der $\mathrm{PaB}$ entzogen. Die eingangs erwähnten Bürgerrechtsgesetze haben $z$ war die Segregationsgesetze teilweise rüdkgängig gemacht; sie enthalten aber auch Paragraphen, die hauptsächlich gegen die politisch engagierten Afro-Amerikaner ge- 
richtet sind und deren rechtliche Lage sehr verschlechtern; diese Bestimmungen werden allgemein Stokely Carmichael-Rap Brown Act genannt:

Hohe Geldstrafen und bis zu fünf Jahren Gefängnis werden allen Personen angedroht, die von einem Bundesstaat zu einem andern reisen, telefonieren oder Briefe schreiben, um zum Aufruhr anzustiften, falls ihnen eine versuchte oder vollendete Beteiligung an solchen *riots * gemeinsamen Gewalttätigkeiten von mindestens drei Personen - wirklich nachgewiesen werden kann. ${ }^{11}$

Da nach Schätzung des New Yorker Guardian allein 1967 ca. 20\% der schwarzen Slumbewohner an solchen Rebellionen teilgenommen haben, stehen den Abhör- und Uberwachungsorganen sowie der Justiz noch große Aufgaben bevor. Die ersten Prozsse nach dem Carmichael-Brown-Acr sind Anfang dieses Jahres angelaufen.

Carmichael selbst scheint nach seiner Hochzeit mit Miriam Makeba ein gewisses Arrangement eingegangen zu sein, um seinen $\mathrm{PaB}$ zurüdkzuerhalten. Auch von Jones heißt es, daß er auf Grund seiner beschwichtigenden Haltung während der Newarker Rebellion I968 Befürworter für das Revisionsverfahren gewonnen habe.

Bei dieser Lage der Dinge ist es dann nicht verwunderlich, wenn der Informationsminister der Black Panthers, der 1935 geborene Eldridge Cleaver, solche Arrangements ebenso scheut wie die ihm drohende Höchststrafe und einen neuen Weg im Umgang mit der Rassenjustiz geht. Er wurde bei einer Polizeiaktion am 6. April r 968 (bei der der 17-jährige Afro-Amerikaner Bobby Hutron von der Polizei erschossen wurde) durch einen Schuß verletzt und steht nunmehr selber unter Anklage des versuchten Mordes an einem weißen Polizisten. Er wurde gegen 50000 Dollars aus der Untersuchungshaft entlassen und zog es vor, zu den Verhandlungen (am 27. November und am 23. Dezember 1968) erst gar nicht mehr zu erscheinen. Sein Aufenthaltsort ist dem weißen Gericht unbelsannt ...

II Frankfurter Allgemeine Zeitung v. I3.4. 1968. 